\title{
Mathematical Modelling of a Wave-Energy Converter
}

William Lee, Michael Castle, Patrick Walsh, Patrick Kelly and Cian Murtagh

\begin{abstract}
We report progress towards developing a mathematical model that can be used to optimise the design of a novel power take off unit for a wave energy generator. We show that the power take off unit can be considered as a non-smooth, dissipative dynamical system. We derive equations of motion using the Lagrangian framework, incorporating a Rayleigh dissipation function and discuss a procedure for generating approximate analytical solutions.
\end{abstract}

\section{Introduction}

Limerick Wave Ltd, are developing a new Power Take Off (PTO) system [1] which they aim to optimise. The design takes alternative movement induced by water waves and converts them into continuous movement via the use of a freewheeling system. In order to give an estimation for the power output of the Wave Energy Converter (WEC), one must derive the equation of motion for the angular velocity of the flywheel for a given set of conditions. See Fig. 1.

Dr William Lee

MACSI, Department of Mathematics and Statistics, University of Limerick, Limerick, Ireland and Department of Mathematics, University of Portsmouth, Portsmouth, UK e-mail: william.lee@port.ac.uk

Michael Castle

Department of Mathematics, University of Portsmouth, Portsmouth, UK e-mail: castlemichae195@gmail.com

Patrick Walsh

Limerick Wave, Limerick, Ireland. e-mail: patrick.walsh@ul.ie

Patrick Kelly

Limerick Wave, Limerick, Ireland. e-mail: patrick.kelly@ul.ie

Cian Murtagh

Seapower, Unit 3, Pier Road, Enniscrone, Co. Sligo, Ireland. e-mail: cian.murtagh@ seapower.ie 
Fig. 1 Wave Energy Converter (WEC) under development by Limerick Wave.

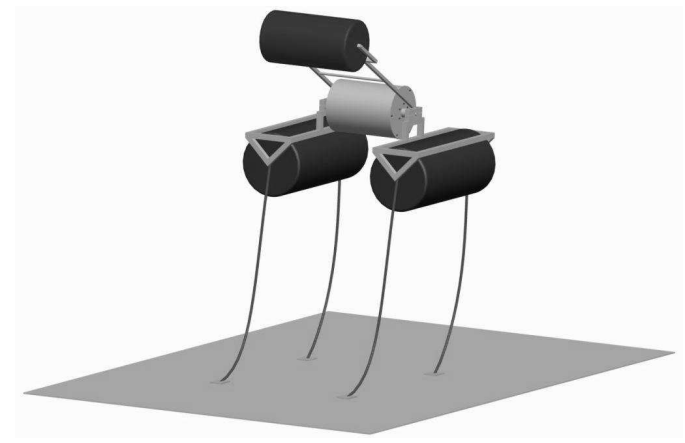

Three modes of motion are to be taken into account: positive sticking, negative sticking, and freewheeling. Positive (Negative) sticking occurs when the fly wheel is being driven by the upward (downward) motion of arm induced by waves. These occur under different conditions and each have their own constraints applied. Dissipative and non-conservative forces act on the system, with non-smooth dynamics due to sprags [4] as well as referred inertia [2].

Analytical solutions are required in order to optimise sizing. The Lagrangian method was used, along with a dissipation function [3] in order to incorporate the non-conserved forces into the model. Although starting with 7 coordinates, one for each of the gears, applying constraints instead bring us to two, with a further reduction available for both positive and negative sticking. See Fig. 2.

Fig. 2 Epicyclic gearing system used in the Power Take Off (PTO). Sprags are indicated by a 's'.

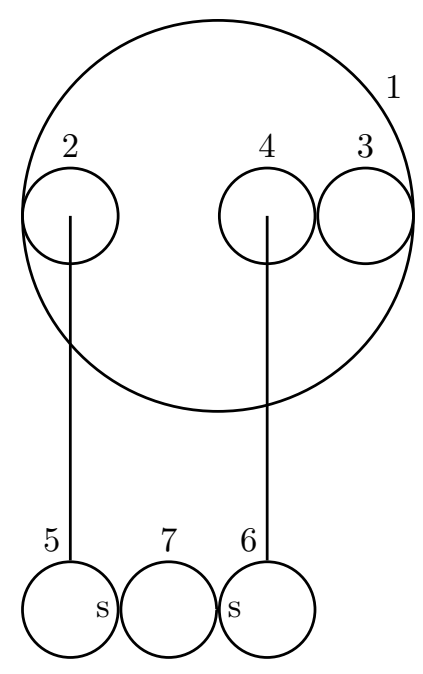

After finding general solutions to the equations of motion for the different states, we must then discover the transition times between the different modes in order to 
perform the correct power integral. The motion is periodic, and hence we need only to describe the motion of the flywheel between 3 transition times.

\section{Equations of Motion}

A derivation of the equations of motion for all three states, including the conditions for transitioning between them are given in this section.

To start, we evaluate the kinetic energies and potential for the conserved forces giving us the Lagrangian

$$
\mathscr{L}=\frac{1}{2}\left(m L^{2}+I_{1}+\frac{I_{2} T_{1}^{2}}{T_{2}^{2}}+\frac{I_{3} T_{1}^{2}}{T_{3}^{2}}+\frac{I_{4} T_{1}^{2}}{T_{4}^{2}}+\frac{I_{5} T_{1}^{2}}{T_{2}^{2}}+\frac{I_{6} T_{1}^{2}}{T_{4}^{2}}\right) \dot{\theta}_{1}^{2}+\frac{1}{2} I_{7} \dot{\theta}_{7}^{2}-\frac{1}{2} k L^{2} \theta_{1}^{2}
$$

where $m$ is the mass of the float, $I_{i}$ is the inertia of gear $i, T_{i}$ is the number of teeth of gear $i, k$ is the spring constant of the float and $L$ is the length of the arm. The Lagrangian incorperates the constraints

$$
\theta_{1}=\frac{\theta_{2} T_{2}}{T_{1}}=\frac{\theta_{3} T_{3}}{T_{1}}=-\frac{\theta_{4} T_{4}}{T_{1}}=\frac{\theta_{5} T_{2}}{T_{1}}=-\frac{\theta_{6} T_{4}}{T_{1}}=\frac{y}{L}
$$

where $\theta_{i}$ is the angular displacement of gear $i$ and $y$ is the vertical displacement of the float, which hold in all three phases.

In order to apply the Lagrangian operator successfully we need to make use of Rayleigh's Dissipation Function [3], $R$, below which allows us to include the nonconservative forces acting on the system. $R$ is given by

$$
R=\frac{1}{2} k \dot{y}^{2}+\tau \dot{\theta}_{7}+L F \cos (\omega t) \dot{y}
$$

The equations of motion of the system are then given by

$$
\frac{d}{d t}\left(\frac{\partial L}{\partial \dot{q}_{i}}\right)-\frac{\partial L}{\partial q_{i}}=Q_{i}
$$

In the case of a freewheeling system this gives us two equations of motion.

$$
I_{7} \ddot{\theta}_{7}=\tau
$$

and

$$
A \ddot{\theta}_{7}+C L^{2} \dot{\theta}_{7}+K L^{2} \theta_{7}=L F \cos (\omega t)
$$

where $A=m L^{2}+I_{1}+\frac{I_{2} T_{1}^{2}}{T_{2}^{2}}+\frac{I_{3} T_{1}^{2}}{T_{3}^{2}}+\frac{I_{4} T_{1}^{2}}{T_{4}^{2}}+\frac{I_{5} T_{1}^{2}}{T_{2}^{2}}+\frac{I_{6} T_{1}^{2}}{T_{4}^{2}}$. Note we need only $\theta_{7}$ in order to determine the motion during this phase. 
Upon applying the additional constraint $\theta_{1}=\frac{T_{4} T_{7}}{T_{1} T_{6}} \theta_{7}$ we achieve the positive sticking equation of motion

$$
A\left(\frac{T_{4} T_{7}}{T_{1} T_{6}}\right) \ddot{\theta}_{7}+C L^{2}\left(\frac{T_{4} T_{7}}{T_{1} T_{6}}\right) \dot{\theta}_{7}+K L^{2}\left(\frac{T_{4} T_{7}}{T_{1} T_{6}}\right) \theta_{7}=L F \cos (\omega t)+\frac{T_{1} T_{6}}{T_{4} T_{7}} \tau
$$

Similarly using $\theta_{1}=-\frac{T_{2} T_{7}}{T_{1} T_{5}} \theta_{7}$ as the additional constraint, we get

$$
A\left(\frac{T_{2} T_{7}}{T_{1} T_{5}}\right) \ddot{\theta}_{7}+C L^{2}\left(\frac{T_{2} T_{7}}{T_{1} T_{5}}\right) \dot{\theta}_{7}+K L^{2}\left(\frac{T_{2} T_{7}}{T_{1} T_{5}}\right) \theta_{7}=L F \cos (\omega t)+\frac{T_{1} T_{6}}{T_{4} T_{7}} \tau
$$

for our negative sticking equation of motion.

Transition times, as well as constants of integration must then be found. The condition for which the flywheel leaves Positive or Negative sticking and instead begins to freewheel is that when the kinetic energy it would have when freewheeling is greater than it would be during a sticking phase. That is, when

$$
\begin{aligned}
& \ddot{\theta}_{7}^{F W}>\ddot{\theta}_{7}^{P S} \\
& \ddot{\theta}_{7}^{F W}>\ddot{\theta}_{7}^{N S}
\end{aligned}
$$

where $\dot{\theta}^{F W}$ is the angular velocity when freewheeling whilst $\dot{\theta}^{P S}$ and $\dot{\theta}^{N S}$ are the angular velocities of positive and negative sticking respectively.

When calculating the transition points these should be interpreted as the values of $\ddot{(\theta})_{7}^{\text {mode }}$ if the system was constrained to be in mode(=FW, PS, NS)

The transition point is that at which equality holds. We must also have continuity of velocity at all transition times. There is a total of four equations of the type

$$
\begin{aligned}
\dot{\theta}_{7}^{F W} & =\dot{\theta}_{7}^{P S} \\
\dot{\theta}_{7}^{F W} & =\dot{\theta}_{7}^{N S}
\end{aligned}
$$

For the final equation, we must have continuity of displacement. Due to the periodic nature, we must have that the path of the velocity ends where it begins, giving us the final equation.

$$
\theta_{7,1}^{F W}=\theta_{7,2}^{F W}
$$

where the subscripts signify the difference between the expressions.

Finally, by integrating the rate of work done against flywheel resistance over a single period, $T$ and averaging, we can calculate the average power generation, $\bar{P}$,

$$
\bar{P}=\frac{1}{T} \int_{0}^{T} \tau \dot{\theta_{7}} d t
$$

A procedure for developing analytic approximations to solutions of these equations has been developed. With the reduced model each of the equations of motion, as well as the transition times can be solved analytically after making use of small 
angle approximations and application of Taylor's expansion formula. The transient restarts at each transition requiring us to only review a small section of the motion.

\section{Results}

Numerical solutions to the equations of motion are shown in Fig. 3. The figure shows $\dot{\theta}_{7}$ and $\dot{\theta}_{1}$ corrected by a factor of the gearing ratio $\frac{T_{1} T_{6}}{T_{4} T_{7}}=\frac{T_{1} T_{5}}{T_{2} T_{7}}$ to allow direct comparison between the angular velocities.

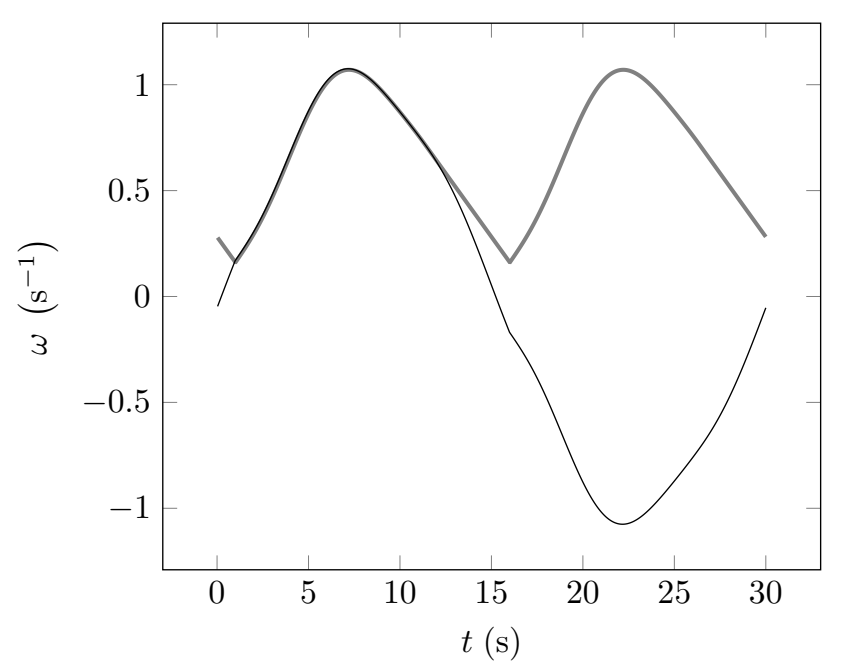

Fig. 3 Angular velocities. Thick grey line: $\dot{\theta}_{7}$ against time. Black line $\dot{\theta}_{1}$ against time (corrected by a gearing ratio to be comparable to $\dot{\theta}_{7}$ ).

\section{Conclusion}

The Limerick Wave PTO is a complex dynamical system with dissipative, nonsmooth dynamics. We have shown that the equations of motion of this system can be modelled using a Lagrangian and a dissipation function. Future work will focus on optimising the power output as a function of design parameters.

\section{Acknowledgements}

WTL acknowledges the support of MACSI, the Mathematics Applications Consortium for Science and Industry (www.macsi.ul.ie), funded by the Science Founda- 
tion Ireland Investigator Award 12/IA/1683. MC acknowledges the funding support from the London Mathematical Society, via its Undergraduate Research Bursaries in Mathematics Scheme, that has contributed to this publication. PW, PK and CM acknowledges the support of Enterprise Ireland via the Innovation Voucher scheme.

\section{References}

1. Burns, Shane, Michael Chapwanya, Cathal Cummins, Paul Dellar, Joe Giddings, Panagiotos Giounanlis, Peter Hicks et al.: Limerick Wave: using flywheel technology to convert the power of the waves to electricity. Technical report $686 \mathrm{http} / /$ www.maths-in-industry.org/miis/ (2013).

2. Gerstmayr, J., and Ambrósio J. A. C.: Component mode synthesis with constant mass and stiffness matrices applied to flexible multibody systems. Int. J. Numer. Methods Eng. 73:15181546 (2008).

3. Goldstein, H., Poole, C., Safko, J.: Classical Mechanics, pp. 22-24. Pearson Education International, Addison Wesley (2002)

4. Richter, H., and Maynard R. E.: Hybrid-dynamical modelling, characterization, and experimental verification of a free-wheeling clutch. Proc. Inst. Mech. Eng. Part I-J Syst Control Eng. 224:361-372 (2010). 